\title{
Usable Secure Email Communications - Criteria and evaluation of existing approaches
}

\author{
Cristian T. Moecke and Melanie Volkamer \\ TU Darmstadt / Center for Advanced Security Research Darmstadt, Germany \\ cristian.moecke@cased.de, melanie.volkamer@cased.de
}

\begin{abstract}
Email communication has been used for many years and replaces more and more traditional letters. Compared to postal service the mail service is easier, faster and free of charge. However, the standard email is from a security point of view comparable to post cards and not to letters. While end-to-end secure email communication is possible with PGP and S/MIME, only few people use it due to a lack of awareness, low usability, and lacking an understanding of PKIs. Recently, some new approaches for secure email communication have been proposed. In order to enable a comparison of all these different email services we define security, usability, and interoperability criteria and apply them to existing approaches. Based on the result, we propose future directives for usable secure email communication.
\end{abstract}

\section{Keywords}

Secure email, Usable Security, Criteria

\section{Introduction}

Ray Tomlinson sent the first network email in 1971 using the ARPANET. With the conversion from ARPANET to the Internet in the early 1980s, email communication became broadly available. Since then, email has become more and more popular. Nowadays, email accounts are free of charge and much faster than traditional letters sent via postal services. Many people have several email accounts. Email replaces traditional letters more and more in many areas including in the private, business, and governmental sector. Critical, sensitive, personal and business information are sent via email although it is well known that emails are less secure than traditional letters. They can easily be forged; and provide neither strong sender authenticity nor message confidentially. Secure email communication is in general available i.e. based on PGP or S/MIME. These techniques are far from being broadly used. Studies like (Whitten, 1999) and (Sheng, 2006) show that these solutions are not usable. People in particular do not understand the concept of standard Public Key Infrastructures (PKI).

Solutions for more usable secure email communication have recently been proposed to improve the situation. Examples are the E-PostBrief (http://www.epost.de), DeMail (https://www.bsi.bund.de) and Hushmail (http://www.hushmail.com/). These approaches do not provide end-to-end secure email communication but users need to trust the service providers. As in addition some of these solutions are closed systems 
and users need to pay for them; it is unclear whether they will be accepted like common emails. As an optimal solution for usable and end-to-end secure email communication is not yet available, users need to find an adequate trade-off for their needs. This paper defines the most relevant criteria that should be taken into account for such a decision. In addition we analyse existing security mechanisms in the context of email communication (namely DKIM, SPF, PGP, S/MIME and TLS) and popular mail service providers (namely Gmail, Hushmail, E-PostBrief and DE-Mail) according to these criteria.

The paper is structured as follows: In Section 2, we analyse related work. In Section 3 we define the security criteria, which are applied to available security techniques in Section 4 and existing email providers in Section 5. In Section 6 we present our conclusions and directions for future work.

\section{Related Work}

There is little published literature that proposes criteria for classifying and analysing the security of an email system. We analyse this work and how it compares to our work: NIST (National Institute of Standards and Technology) published Guidelines on Electronic Mail Security (NIST, 2007). The guide "is intended to assist organizations in installing, configuring, and maintaining secure mail servers and mail client” (NIST, 2007). The guide is based on available technology and on assisting administrator and users to apply this technology in the most secure way. Our work proposes criteria independent from available email systems and technologies. Alperovitch et al. (Alperovitch et al., 2007) analyses some of the previous work on email reputation systems (systems that calculate a score for an email, usually for spam filtering) and provide a "taxonomy that examines the required properties of email reputation systems, identifies the range of approaches, and surveys previous work" (Alperovitch et al., 2007). While their work classifies and organizes a broad range of reputation criteria, they do not analyse whether current systems ensure these criteria. Garfinkel (Garfinkel, 2005b) focuses on usability aspects of email security, and includes a survey on how users act regarding signed emails. There are also previous works on usable security analysis of secure email systems (Whitten, 1999) (Garfinkel, 2005a) (Sheng, 2006), which provide important usability criteria, which we also consider on our work.

\section{Email usable security criteria}

This section defines the evaluation criteria used to later analyse existing email security techniques and email providers. They include security, interoperability, and usability requirements. Due to space constraints, we leave aspects like malware spreading, spam, traceability, long-term authenticity and confidentiality, legal aspects and anonymous communication including aliases for future work.

Security: The security properties are divided into sender authenticity (A), integrity $(\mathrm{I})$, confidentiality $(\mathrm{C})$, and one requirement addressing trust in the service under evaluation (Tr). These criteria are deduced from our threat analysis of different 
email communication scenarios and possible attacks. We distinguish the following requirements: (A.1) It should be possible to verify that the sender is the owner of the email account that belongs to the stated sender email address. (A.2) It should be analysed which methods are used to verify the authenticity of the user i.e. password, two-factor authentication, or asymmetric cryptography (This authenticity information, especially how strong the authentication method was, are of interest to the receiver of a message. However, none of the later evaluated approaches provide this kind of information to the receiver.). This also includes whether passwords are securely transmitted or not. (A.3) It should be possible to deduce the real identity of the person sending the email. (I) It should be possible to detect modification of the content/the subject of the received email. (C) No one else except the receiver of the email should gain access to the content/subject of the email. (Tr) It should be analysed whether the user needs to trust the email provider or any other party like certification authorities regarding any of the security requirements defined previously.

Interoperability: Interoperability is important, as services are not ready for largescale usage without it. Correspondingly, the sub criteria are related to security and trust aspects that are important for the acceptance of the corresponding service. This class of criteria is divided into the following sub criteria (Note, not all requirements are addressed here. Another one for instance is that the email service should provide all the functionality provided by standard email like searching in the inbox or accessing the account from different devices.): (IN.1) It should be possible to communicate with people who do not use the same email provider. (IN.2) It should be possible to securely communicate with people who do not use the same email provider. (IN.3) It should be possible to setup and run your own email service. (IN.4) There should be more than one provider offering the corresponding service. (IN.5) It should be possible to use the system with the security techniques discussed in Section 4.

Usability aspects: The usability criteria are deduced from papers on usable security analysis of email communication systems. The most important are: (U.1) It should be evaluated how much work is necessary to setup and start using the service. (U.2) Adequate metaphors should be used for functions like encryption, signing, and certificates. (U.3) It should only require less and easy to make trust decisions. (U.4) Sender and receiver should not need to understand the underlying PKI concepts to make any trust decisions. (U.5) It should not be necessary to obtain and/or compare some information via out of bounds mode like comparing hashes.

\section{Security techniques available for email}

In this section we describe the security techniques that are currently available and could be used to improve email security by integrating them in existing email services or by installing a corresponding plugin into the email client or web browser. The list contains techniques related to sender authentication, Public Key Infrastructure (PKI) support, and securing the network. We analyse which of the above security and usability criteria they fulfil while we only discuss those security requirements that are at least partially ensured and only those usability requirements 
that can be applied but are not fulfilled. We also do not address interoperability requirements as the analysed techniques do not stand on their own but can only be used as an add-on to the email services discussed in Section 5. For all techniques we make the trust assumption ( $\mathrm{Tr}$ ) that the software running the corresponding security operations and checks is trustworthy, and that cryptographic secret keys are securely stored.

An SPF (Sender Policy Framework) (RFC4408) record is a list of IP addresses that are authorized to send emails for a particular domain. The DNS server responsible for this domain publishes this list. This list can be used to partially verify the authenticity of a received email, confirming that it came from an authorized server. If we trust the DNS server regarding the integrity of this list and the email provider behind this domain ( $\mathrm{Tr}$ ), i.e. the provider only delivers emails from authenticated users with the correct address in the "from" field, and there is also no MITM attack (A Man-In-The-Middle attack could modify the list since it is not authenticated.) when fetching the list, A.1 is ensured. However since this assumption is not true for all DNS servers and all mail providers, hard trust decisions remain for the user (U.3). Sender ID Framework - SIDF (RFC4406) extends the SPF verification, but with no relevant improvements considering our criteria. DKIM (Domain Keys Identified Mail) (RFC4871) is a digital signature from the email service provider on sent emails. It allows verifying that this provider really sent the message. The corresponding public key is published on the DNS server associated to this domain. Thus, regarding A.1, one needs to trust that the DNS server provides the proper key and that the email service only delivers emails from authenticated users. Correspondingly, the trust assumptions (Tr) regarding A.1 and the limitations for U.3 identified for SPF also hold for DKIM, including the non-occurrence of a MITM attack (In this case the MITM attack could be used to inform the wrong key to the receiver). In addition, the integrity property (I) is partially ensured because the signature provides message integrity after being processed by the mail server of the sender under the same trust assumption required for A.1.

PGP (Pretty good Privacy) (RFC2015) provides end-to-end security to email messages. It is not necessary to trust a central trust anchor as it is based on the Webof-Trust (WoT) model. It fulfils property C and I. Authenticity depends on the adequate use of the WoT. In a restricted environment where people verify carefully the keys they sign, A.1 and even A.2 can be considered as fulfilled, but with the cost of hard trust decisions (conflict with U.3) and the fact that out-of-bounds data (i.e. verifying key hashes before setting keys as trusted) is required (conflict with U.5). PGP requires some PKI understanding (conflict with U.4) and it takes a while to get started (conflict with U.1). Adequate metaphors (U.2) are very important for a user friendly implementation of PGP, while studies (Whitten, 1999) (Garfinkel, 2005a) showed that this is not the case for PGP (conflict with U.2). S/MIME (Secure/Multipurpose Internet Mail Extensions) (RFC2311) provides end-to-end security to email messages. It is based on X.509 PKIs. A certificate for a corresponding key pair should be obtained from a trusted Certification Authority (CA) which is usually not free of charge. However, the use of self-signed certificates is also possible. S/MIME with CAs fulfils A.1, C and I, and if the CA also verifies the personal identity (i.e. for Qualified Certificates on E.U. (RFC3739)) it fulfils A.2, 
as well. The receiver needs to trust the CA that issued the certificate of the sender (Tr). While major CAs are already set as trusted on most plugins, it leads to hard trust decisions if the sender's certificate is issued on an untrusted/unknown CA (conflict with U.3). Without CAs (self-signed certificates), S/MIME only fulfils C and I. In this case it is hard to decide if the authenticity of the message should or should not be trusted (conflict with U.3). The user needs out-of-bounds information to verify the authenticity (conflict with U.5). In both cases, it takes a while to get started (conflict with U.1). Similar to PGP, adequate metaphors are very important for a user-friendly implementation. In addition, in cases in which the verification fails (i.e. failure on obtaining revocation information, or an intermediate CA certificate), it is required to understand the underlying PKI (conflict with U.4).

Opportunistic Encryption (RFC4322) in general means that a system tries to use encryption to secure any communication, while using unencrypted communication if corresponding keys are not available. The keys are not necessarily authenticated. Thus, if there is any known key to communicate with the receiver (even in a selfsigned certificate) encryption is used. Garfinkel (Garfinkel, 2003) proposed a system that implements opportunistic encryption for email communication. It acts like a proxy for sending and a proxy for receiving an email (While he does not provide clear information about the proxy, we assume that it runs as a plugin locally and is considered trusted (Tr).). The proxy manages the keys (including certificates), the encryption and the decryption process. Whenever the sending proxy is able to encrypt an email to the receiver, it will encrypt this message. It is also possible to specify that a message should only be delivered if it can be encrypted. There is no authentication of the keys in the system. The receiver proxy learns new keys from emails previously received and stores them in its own database. It gives a warning only if a new key is detected for an already-known email address. Furthermore, emails are not signed. It therefore provides confidentiality (C) under the trust assumption ( $\mathrm{Tr}$ ) that the proxy is trustworthy (i.e. a local proxy) and there was a previous legitimate communication between sender and receiver so the correct key is known. This idea avoids the need to understand PKI concepts (U.4 is ensured) and hard trust decisions (U.3 and U.5 is in general ensured - except when a conflict occurs). There is also a low setup effort (U.1).

TLS (Transport Layer Security) provides a secure communication between user and server (over IMAP, POP, SMTP or a Webmail session) and between servers. Confidentiality (C) and integrity (I) are ensured under the trust assumption ( $\mathrm{Tr}$ ) that the whole delivery process is secured by TLS and that the servers are trustworthy. It could also be used to authenticate the user with TLS client authentication (therefore offering strong authentication - A.2), but that is not common.

\section{Analysis of existing secure email providers}

In this section, we analyse five types of email providers according to our criteria while focusing on one representative of each; namely standard (in-secure) email providers, very popular email providers providing some security (Gmail), a combination of the previous and the different PKI approaches from Section 4, providers offering secure email communication in a closed system (DE-Mail/E- 
Postbrief), and providers offering secure email communication in an open system (Hushmail). The results of the comparison are summarized in Tables 1, 2 and 3.

\begin{tabular}{|c|c|c|c|c|c|}
\hline & A.1 & A. 2 & A.3 & $\mathrm{I}$ & $\mathrm{C}$ \\
\hline Gmail & $\begin{array}{|lr|}\text { Partially (see U.3 } \\
\text { Tr: Gmail/DNS). } \\
\text { Detect forged } \\
\text { Gmail(Tr: Gmail) }\end{array}$ & $\begin{array}{lr}2 \quad \text { factor } & \text { (not } \\
\text { visible } & \text { to } \\
\text { receiver) } & \\
\end{array}$ & No & $\begin{array}{l}\text { Yes (Tr: Gmail } \\
\text { and DNS) }\end{array}$ & $\begin{array}{lr}\text { End to Server } \\
\text { and Server } & \text { to } \\
\text { Server } & \text { (if } \\
\text { available) } & \\
\end{array}$ \\
\hline DE-Mail & $\begin{array}{ll}\text { Yes } & \text { (Tr: } \\
\text { Provider) } & \\
\end{array}$ & Unl & $\begin{array}{ll}\text { Yes } & \text { (Tr: } \\
\text { Provider) } & \\
\end{array}$ & $\begin{array}{ll}\text { Yes } & (\mathrm{Tr}: \\
\text { Provider }) & \\
\end{array}$ & $\begin{array}{ll}\text { Yes } & \text { (Tr: } \\
\text { Provider) }\end{array}$ \\
\hline $\begin{array}{l}\text { E- } \\
\text { PostBrief }\end{array}$ & $\begin{array}{ll}\text { Yes } & \text { (Tr: } \\
\text { Provider) }\end{array}$ & $\begin{array}{l}\text { SMS TAN (not } \\
\text { visib. to receiver) }\end{array}$ & $\begin{array}{ll}\text { Yes } & \text { (Tr: } \\
\text { Provider) } & \\
\end{array}$ & $\begin{array}{ll}\text { Yes } & \text { (Tr: } \\
\text { Provider) } & \\
\end{array}$ & $\begin{array}{ll}\text { Yes } & \text { (Tr: } \\
\text { Provider) } & \\
\end{array}$ \\
\hline Hushmail & $\begin{array}{l}\text { Yes } \\
\text { Hushmail) } \\
\end{array}$ & Password only & No & $\begin{array}{lr}\text { Yes } & (\mathrm{Tr}: \\
\text { Hushmail/Softw.) }\end{array}$ & $\begin{array}{lr}\text { Yes } & \text { (Tr: } \\
\text { Hushmail/Softw.) }\end{array}$ \\
\hline $\begin{array}{l}\text { Gmail \& } \\
\text { PGP }\end{array}$ & $\begin{array}{l}\text { Restricted } \\
\text { contexts (no U.5) }\end{array}$ & $\mathrm{N}$ & $\begin{array}{l}\text { Restricted } \\
\text { contexts (no U.5) }\end{array}$ & Yes & Yes \\
\hline $\begin{array}{l}\text { Gmail \& } \\
\text { S/MIME }\end{array}$ & $\begin{array}{l}\text { If CA verifies it } \\
\text { (Tr: CA) }\end{array}$ & & $\begin{array}{l}\text { If CA verifies it } \\
\text { (Tr: CA) }\end{array}$ & Yes & Yes \\
\hline $\begin{array}{l}\text { Gmail \& } \\
\text { Opp }\end{array}$ & $\begin{array}{l}\text { If had previous } \\
\text { secure comm. }\end{array}$ & & No & No & $\begin{array}{l}\text { If receiver key is } \\
\text { known }\end{array}$ \\
\hline
\end{tabular}

Table 1 - E-mail services and security criteria

\begin{tabular}{|c|c|c|c|c|c|}
\hline & U.1 & U.2 & U.3 & U.4 & U.5 \\
\hline Gmail & Easy & No 1 & $\begin{array}{l}\text { Decide if domain } \\
\text { is trustworthy }\end{array}$ & No PKI & $\begin{array}{l}\text { Domain } \\
\text { trustworthiness }\end{array}$ \\
\hline DE-Mail & $\begin{array}{l}\text { Identification on } \\
\text { provider }\end{array}$ & & $\begin{array}{ll}\text { Easy: } & \text { Trust } \\
\text { provider } & \\
\end{array}$ & PKI hidden & $\begin{array}{l}\text { No out of bounds } \\
\text { data }\end{array}$ \\
\hline $\begin{array}{l}\text { E- } \\
\text { PostBrief }\end{array}$ & $\begin{array}{l}\text { Identification on } \\
\text { Post office }\end{array}$ & $\begin{array}{l}\text { Encryption } \\
\text { Signature }\end{array}$ & $\begin{array}{ll}\text { Easy: } & \text { Trust } \\
\text { provider } & \\
\end{array}$ & PKI hidden & $\begin{array}{l}\text { No out of bounds } \\
\text { data }\end{array}$ \\
\hline Hushmail & Easy & $\begin{array}{l}\text { Encryption } \\
\text { Signature }\end{array}$ & $\begin{array}{ll}\text { Easy: } & \text { Trust } \\
\text { provider } & \\
\end{array}$ & PKI hidden & $\begin{array}{l}\text { No out of bounds } \\
\text { data }\end{array}$ \\
\hline $\begin{array}{l}\text { Gmail \& } \\
\text { PGP }\end{array}$ & $\begin{array}{l}\text { Hard: create } \\
\text { /publish key, get } \\
\text { recipient key }\end{array}$ & $\begin{array}{l}\text { Depends } \\
\text { imple- ment }\end{array}$ & $\begin{array}{l}\text { Decide trusted } \\
\text { keys based on } \\
\text { WoT }\end{array}$ & Understand PGP & $\begin{array}{l}\text { Obtain } \\
\text { information } \\
\text { trust keys }\end{array}$ \\
\hline $\begin{array}{l}\text { Gmail \& } \\
\text { S/MIME }\end{array}$ & $\begin{array}{l}\text { Hard: obtain } \\
\text { own/recipient } \\
\text { certificate }\end{array}$ & $\begin{array}{l}\text { Depends on } \\
\text { imple-mentation }\end{array}$ & $\begin{array}{l}\text { If known CA, } \\
\text { easy. If not, hard }\end{array}$ & $\begin{array}{l}\text { If verification } \\
\text { fails or CA is } \\
\text { untrusted }\end{array}$ & $\begin{array}{l}\text { If verification } \\
\text { fails or CA is } \\
\text { untrusted }\end{array}$ \\
\hline $\begin{array}{l}\text { Gmail \& } \\
\text { Opp }\end{array}$ & Easy & Not necessary & $\begin{array}{l}\text { Only in key } \\
\text { conflict }\end{array}$ & PKI hidden & $\begin{array}{l}\text { Only in key } \\
\text { conflict }\end{array}$ \\
\hline
\end{tabular}

Table 2 - E-mail services and usability criteria

\begin{tabular}{|l|l|l|l|l|l|}
\hline & IN.1 & IN.2 & IN.3 & IN.4 & IN.5 \\
\hline \hline Gmail & Yes & Yes & Open standards & Open standards & PGP, S/MIME \\
\hline DE-Mail & Closed environ. & Closed environ. & High cost & High cost & PGP/S/MIME \\
\hline E-PostBr. & Closed environ. & Closed environ. & \multicolumn{1}{|l}{ Closed environ. } & No & PGP/S/MIME \\
\hline \hline Hushmail & Yes & Yes & Open standards & Open standards & $\begin{array}{l}\text { DKIM/SPF/ } \\
\text { S/MIME }\end{array}$ \\
\hline
\end{tabular}

Table 3 - E-mail services and interoperability criteria

\subsection{Standard email}

According to the email standards (RFC5322, RFC5321), standard email services are not required to provide too much security information to the receiver. The only 
somehow relevant security information is the header of an email which provides information regarding the delivery path of this message, but this information is not authenticated, therefore any decision based on it depends on assuming all the servers on the path trustworthy. However these assumptions are hard to verify and rely on too many out-of-bounds information (conflict with U.5) and hard trust decisions (conflict with U.3), and also are not true for many servers. Many email providers operate using no more security than those offered by these standards.

\subsection{Gmail}

The most popular email providers usually offer at least some security improvements, even if mainly for spam control. We have chosen to analyse Gmail, which verifies SPF and DKIM for email origin authentication, and adds DKIM signatures for emails delivered. When an email has SPF or DKIM authentication but the domain in the address ("from" field) does not match the authenticated origin information, this is shown to the end user as a "via authenticated origin domain" after the sender's address. Emails sent from forged Gmail accounts (i.e. from other servers, but with Gmail address on the "from" field), which as a result do not have authenticated information on their origin, are shown to the receiving Gmail user with a warning "this message may not have been sent by: email@domain.com”, in red). This makes impersonating other Gmail accounts difficult.

Emails from domains other than Google and without SPF and without DKIM authentication are shown without any hint or warning. The only difference between a non-authenticated and an authenticated mail is that the authenticated domain is shown as "mailed-by" when the user looks at the details of the message header. There is no other visual clue that differentiates an SPF/DKIM authentic message from one without any authentication. Therefore, Gmail only guarantees email proof of possession (A.1) between Gmail users, considering Gmail trustworthy (Tr). When communicating with other service providers, the interface does not show adequate feedback, but even with more information provided it would lead to complex trust decisions (conflicts with U.3), namely deciding if the authenticated domain is trustworthy or not. Gmail supports two-factor authentication (using a smartphone application that generates "One Time Passwords") but this is not enforced (A.2) and not visible to the receiver. Emails originated from Gmail servers are DKIM signed, therefore have their integrity guaranteed (I), considering Gmail trustworthy (Tr). Gmail uses SSL/TLS for end-to-server encryption, and may use it also to communicate with other servers when supported, however even trusting Gmail it is not possible to guarantee confidentiality of messages after they leave Gmail servers. Therefore, (C) is only partially fulfilled. The communication between user and server is encrypted during the authentication process (A.2).

Gmail uses only open standards and is a standard mail provider (IN.1). All the security measures available are also based on open standards and may be used by other providers, so it is possible to have the same level of secure email communication with any other interested provider (IN.2). While there is only one provider of the Gmail solution, we consider IN.4 and IN.5 fulfilled because there is no restriction to operate a similar service once it is based on open standards. It is also 
possible to integrate PGP and or S/MIME on Gmail (there are even plugins available for that - i.e. FireGPG and Penango), therefore IN.5 is also fulfilled.

\subsection{DE-Mail/E-PostBrief}

DE-Mail and E-PostBrief are closed messaging systems, provided by the German government and German postal services. These approaches claim to provide secure email communication and are based on strictly controlled servers that manage the full delivery process. There is also a law regulating DE-Mail. Both systems are closed environments. Thus there is no possibility to forge a message (A.1), if the providers are considered trustworthy (Tr). Users are only allowed to use the system after a personal identification (A.3) at the provider's office (DE-Mail) or at a postal service centre (E-PostBrief). E-PostBrief provides SMS TAN (a one-time-password sent via SMS) as a second factor of authentication, but its use is neither enforced (A.2) nor available to the receiver. There is no information available whether DEMail providers will offer this possibility. The communication between different servers of one provider and servers from different providers (in particular within the DE-Mail concept) are secured by TLS. TLS is also used to provide end-to-server security. Correspondingly, integrity (I) and confidentiality (C) are fulfilled based on trust in the provider(s) (Tr). Besides the costs for each email (both services require payment per message sent), interoperability is the greatest disadvantage of these two approaches. Due to their closed environment property, it is only possible to communicate with people who have an account in this environment (IN.1 and IN.2 are not ensured). While there are some providers in DE-Mail, the number is limited number (IN.4 insured) though it is possible to operate your own provider (with high costs involved - IN.3 ensured). It could be possible to use other techniques (IN.5), particularly PGP and S/MIME to provide end-to-end security, using corresponding plugins.

\subsection{Hushmail}

Hushmail is a web-based solution (It is also possible to use a Java applet to download the encrypted key and process the encryption operations locally. However the same trust assumptions hold for the Java version, since Hushmail provides it. Hushmail is also testing a new interface, but it is not stable. Therefore our evaluation considers the old ("original”) interface.). Hushmail calls itself a PGP based secure email system. However, the main characteristic of PGP, namely the WoT model, is not applied. Instead, Hushmail signs all the public keys of the users and publishes them on a key server. Obviously, all keys signed by Hushmail are considered trustworthy. With this approach, it is possible to automate the key trust management process and in particular the user is no longer involved. A Hushmail server generates the user's key pairs and the secret key is encrypted with the user email account password. In addition, everyone is able to publish new PGP keys on the key server, associated to email addresses from other email providers besides Hushmail. In that case, there is a challenge-response mail sent to the email associated with the key to verify the ownership of the account before the key is set as trusted for all Hushmail users. It is also possible to send secure emails to non-Hushmail accounts for which 
no PGP key is stored on the Hushmail key server, based on Question/Answer that only the receiver should be able to answer.

For all received signed emails for which the corresponding public key is trusted by Hushmail, the signature is verified and if the email is properly signed the email is shown as having valid signatures ("This message is encrypted, and is digitally signed by Sender Name <username@domain.com>”), which fulfils A.1. Emails are not verified using DKIM or SPF. There is only a warning if the "return-path" field does not match the sender address. Since this information may be forged, there are no other security indicators of authenticity for non-signed emails that are received. The user's identity is not verified before creating an account (A.3 fails) and the user is authenticated by password.

Email integrity (I) and confidentiality (C) are in general (Due to space constraints we only consider the case where PGP is in place while there are some usability problems with the implementation of the Question/Answer based encryption.) ensured under the assumption that Hushmail is trustworthy (Tr). It is possible for the user to verify message integrity also using its own trusted software and verifying the keys using out-of-bounds data. Since it operates using open standards and can send standard insecure email, all interoperability criteria are fulfilled. The main usability problem is the lack of adequate metaphors for cryptographic operations (U.2) and the communication with users without Hushmail account.

\section{Discussion and conclusions}

In this paper, we proposed criteria, which a usable and secure email system should ideally ensure. The list only contains the most important security and usability requirements due to space limit, but more should be considered on the development of an ideal solution. We applied these criteria to email security techniques and also to existing email providers while discussing different groups of email providers including a combination with available security techniques as add-on. None of them ensures all the requirements.

Closed and web-based systems like Hushmail are more usable, but they do not provide end-to-end security, as one needs to trust the provider. On the other hand, the consequences of solutions based on security add-on for standard email services are hard trust decisions for the user. It also takes a while to set up these systems. In addition, trust in this security technique is also required, as the average user cannot develop this on its own. The Opportunistic Encryption approach is also more usable, however it does not offer authentication. Approaches similar to Hushmail also have the benefit that secure email can be used from any computer while the PGP/SMIME add-ons require you to carry the key in a secure way. The main disadvantage of DEMail/EPostbrief is that these are closed systems, i.e. it is not possible to communicate electronically with people who do not have such an account, and you need to pay for each email. While with Hushmail it is possible to communicate with people who do not have Hushmail both in a secure and insecure way, there are still some security and usability problems in the implementation. 
For future work, we propose to merge these approaches. Combine systems like Hushmail with Opportunistic Encryption to hide the PKI and other security trust decisions even more from the user. Registration should be based on in person identification and authentication. Authentication should be based on two-factor authentication at the least. The type of authentication used by the sender should be provided to the receiver. While the key should be generated at the user's side, the email provider could store the encrypted key to make it accessible from any computer. Key management, distribution, and revocation could be based on S/MIME, but operated by the mail servers rather than a third party CA. Communication to users without such an account could be based on an improved implementation of the Hushmail approach.

The biggest challenge seems to be on usability aspects, especially on trust decisions that the receiver needs to take. Future work should consider this carefully and reduce the necessary trust decisions. The software on the client side must be prepared to offer clear information about the security and trustworthiness of an email, so the user can decide how to react to the content of the message. The same applies for sending a message, the sender should be provided with enough information about how secure the delivery process will be.

The proposed criteria focus on aspects that may facilitate the decision of the receiver about the trustworthiness of an email. If we are able to securely authenticate the origin of an email, we can also use this information for spam filtering and also to display more precise and contextualized warnings e.g. regarding phishing emails and dangerous attachments.

\section{References}

Allman, E., Callas, J., Delany, M., Libbey, M., Fenton, J., Thomas, M.: DomainKeys Identified Mail (DKIM) Signatures. RFC 4871 (Proposed Standard) (May 2007), http://www.ietf.org/rfc/rfc4871.txt, updated by RFC 5672

Alperovitch, D., Judge, P., Krasser, S.: Taxonomy of email reputation systems. In: Distributed Computing Systems Workshops, 2007. ICDCSW'07. 27 $7^{\text {th }}$ International Conference on. pp. 27. IEEE (2007).

Butterfield, J., Tracy, M., Jansen, W.: Guidelines on Electronic Mail Security Recommendations of the National Institute of Standards and Technology (2007)

Dusse, S., Hoffman, P., Ramsdell, B., Lundblade, L., Repka, L.: S/MIME Version 2 Message Specification. RFC 2311 (Historic) (Mar 1998)

Elkins, M.: MIME Security with Pretty Good Privacy (PGP). RFC 2015 (Proposed Standard) (Oct 1996)

Garfinkel, S.: Enabling email confidentiality through the use of opportunistic encryption. In: Proceedings of the 2003 annual national conference on Digital government research. pp. 1-4. Digital Government Society of North America (2003) 
Garfinkel, S., Margrave, D., Schiller, J., Nordlander, E., Miller, R.: How to make secure email easier to use. In: Proceedings of the SIGCHI conference on human factors in computing systems. pp. 701-710. ACM (2005)

Garfinkel, S., Miller, R.: Johnny 2: a user test of key continuity management with S/MIME and Outlook Express. In: Proceedings of the 2005 symposium on Usable privacy and security. pp. 13-24. ACM (2005)

Klensin, J.: Simple Mail Transfer Protocol. RFC 5321 (Draft Standard) (Oct 2008)

Lyon, J., Wong, M.: Sender ID: Authenticating E-Mail. RFC 4406 (Experimental) (Apr 2006)

Resnick, P.: Internet Message Format. RFC 5322 (Draft Standard) (Oct 2008)

Richardson, M., Redelmeier, D.: Opportunistic Encryption using the Internet Key Exchange (IKE). RFC 4322 (Informational) (Dec 2005)

Santesson, S., Nystrom, M., Polk, T.: Internet X.509 Public Key Infrastructure: Qualified Certificates Profile. RFC 3739 (Proposed Standard) (Mar 2004)

Sheng, S., Broderick, L., Hyland, J., Koranda, C.: Why Johnny still can't encrypt: evaluating the usability of email encryption software. In: Symposium On Usable Privacy and Security. pp. 3-4 (2006)

Whitten, A., Tygar, J.: Why Johnny Can't Encrypt. In: USENIX Security. vol. 1999, p. 1 (1999)

Wong, M., Schlitt, W.: Sender Policy Framework (SPF) for Authorizing Use of Domains in EMail, Version 1. RFC 4408 (Experimental) (Apr 2006) 